\title{
REFERENCES
}

1. M. Heins, Riemann surfaces of infinite genus, Ann. of Math. (2) 55 (1952), 296-317.

2. W. Hurewicz and H. Wallman, Dimension theory, Princeton Univ. Press, Princeton, N. J., 1948; Chapter II.

3. Z. Kuramochi, On the behaviour of analytic functions on abstract Riemann surfaces, Osaka Math. J. 7 (1955), 109-127.

4. T. Kuroda, On analytic functions on some Riemann surfaces, Nagoya Math. J. 10 (1956), 27-50.

5. K. Noshiro, Cluster sets, Chapter IV, §2, Springer, Berlin, 1960.

6. N. Toda and K. Matsumoto, Analytic functions on some Riemann surfaces, Nagoya Math. J. 22 (1963), 211-217.

Tokyo Institute of Technology, Tokyo, JAPAN

\section{ON THE EIGENVALUES OF A MATRIX WHICH COMMUTES WITH ITS DERIVATIVE}

\author{
NICHOLAS J. ROSE
}

Let $V(t)$ be an $n \times n$ matrix whose elements are differentiable functions of $t$. Epstein [1] has obtained (Theorem 1) necessary and sufficient conditions for $V(t)$ to commute with its derivative $\dot{V}(t)$ in some interval provided that the Jordan canonical form of $V(t)$ maintains the same form throughout the interval (see the definition below). Using this result we show in Theorem 2, under the same restriction, that if $V(t)$ commutes with $\dot{V}(t)$, then the eigenvalues of $\dot{V}(t)$ are the derivatives of the eigenvalues of $V(t)$.

Definition. Let $S(J)$ be the set of all $n \times n$ matrices $V(t)$ defined in the interval $I: t_{1} \leqq t \leqq t_{2}$ and having the properties:

(i) the elements $V_{i j}(t)$ of $V(t)$ are differentiable functions in $I$,

(ii) for each $V(t) \in S(J)$ there exists a nonsingular differentiable matrix $P(t)$ such that $V=P^{-1} J P$ for $t \in I$ where $J$ is the Jordan canonical matrix

$$
J=\left(\begin{array}{cccccc}
C_{1}(t) & 0 & \cdot & \cdot & \cdot & 0 \\
0 & C_{2}(t) & \cdot & \cdot & \cdot \\
\cdot & \cdot & \cdot & \cdot & 0 \\
0 & \cdot & 0 & & C_{r}(t)
\end{array}\right)
$$

Presented to the Society, April 21, 1964; received by the editors February 17, 1964 and, in revised form, April 22, 1964. 
and

$$
C_{k}(t)=\lambda_{k}(t) I_{k}+E_{k}, \quad k=1,2, \cdots, r
$$

where $C_{k}(t)$ is an $n_{k} \times n_{k}$ matrix, $n_{k}$ being the multiplicity of the eigenvalue $\lambda_{k}(t)$ of $V ; I_{k}$ is the $n_{k} \times n_{k}$ identity matrix and $E_{k}$ is an $n_{k} \times n_{k}$ matrix all of whose elements equal 0 except possibly on the superdiagonal where some elements may equal 1 ,

(iii) $E_{k}$ and $n_{k}$ are constant for $t \in I$.

(iv) $\lambda_{k}(t)$ are differentiable in $I$,

(v) if $j \neq k$ then $\lambda_{j}(t)-\lambda_{k}(t) \neq 0$ for $t \in I$.

In the following $[X, Y]$ stands for the Lie bracket: $[X, Y]=X Y$ $-Y X$.

Theorem 1 (Epstein [1]). If $V(t) \in S(J)$ and $V=P^{-1} J P$ then $[V, \dot{V}]=0$ in $I$ if and only if

$$
\left[J,\left[J, \dot{P} P^{-1}\right]\right]=0, \quad t \in I .
$$

Proof. Setting $X=\dot{P} P^{-1}$ we have by direct calculation

$$
\begin{aligned}
V & =P^{-1}(J+[J, X]) P, \\
{[V, \dot{V}] } & =P^{-1}([J, J]+[J,[J, X]]) P .
\end{aligned}
$$

Since $[J, \dot{J}]=0$, the theorem follows.

We need the following lemma (see Jacobson [2]).

LEMMA. If $A$ is an $n \times n$ matrix and $X$ is any solution of

$$
[A,[A, X]]=0
$$

then $[A, X]$ is nilpotent.

Theorem 2. If $V(t) \in S(J)$ and $[V, \dot{V}]=0$ in $I$, then the eigenvalues of $\dot{V}(t)$ are the derivatives of the eigenvalues of $V(t)$ for $t \in I$.

Proof. If $V=P^{-1} J P$ then from Theorem $1, J$ must satisfy

$$
[J,[J, X]]=0
$$

where $X=\dot{P} P^{-1}$. We partition $X$ in accordance with (1), that is $X=\left(X_{i j}\right)$ where $X_{i j}$ is of order $n_{i} \times n_{j}$. Using (2) we see that $X_{i j}$ must satisfy

$$
C_{i}^{2} X_{i j}-2 C_{i} X_{i j} C_{j}+X_{i j} C_{j}^{2}=0, \quad i, j=1,2, \cdots, r .
$$

The left-hand side of (8) defines a linear operator $T$ acting on $X_{i j}$. The eigenvalues of $T$ are $\left(\lambda_{i}-\lambda_{j}\right)^{2}$ (see Hausner [3]). Therefore if $i \neq j$ the only solution of (4) is $X_{i j}=0$. Consequently $X$ consists only 
of diagonal blocks $X_{i i}$ which satisfy

$$
\left[E_{i},\left[E_{i}, X_{i i}\right]\right]=0, \quad i=1,2, \cdots, r .
$$

It follows from the Lemma that $\left[E_{i}, X_{i i}\right]$ is nilpotent for $i=1, \cdots, r$. We note that the matrix $[J, X]$ consists only of the nilpotent diagonal blocks $\left[E_{i}, X_{i i}\right]$. From (3) we see that $\dot{V}$ has the same eigenvalues as $\dot{J}+[J, X]$, namely the roots of

$$
\operatorname{det}([J, X]+J-\lambda I)=0 .
$$

Since $[J, X]$ consists only of the diagonal blocks $\left[E_{i}, X_{i i}\right]$ and $\dot{J}$ consists only of the diagonal blocks $\dot{\lambda}_{i}(t) I_{i}$, we have

$$
\begin{aligned}
\operatorname{det}([J, X]+J-\lambda I) & =\prod_{i=1}^{r} \operatorname{det}\left(\left[E_{i}, X_{i i}\right]+\left(\dot{\lambda}_{i}-\lambda\right) I_{i}\right) \\
& =\prod_{i=1}^{r}\left(\dot{\lambda}_{i}-\lambda\right)^{n_{i}}
\end{aligned}
$$

since $\left[E_{i}, X_{i i}\right]$ is nilpotent. Therefore the eigenvalues of $\dot{V}$ are just $\dot{\lambda}_{i}$ and the theorem is proved.

\section{REFERENCES}

1. I. J. Epstein, Conditions for a matrix to commute with its integral, Proc. Amer. Math. Soc. 14 (1963), 266-270.

2. N. Jacobson, Lie algebras, Interscience, New York, 1962.

3. M. Hausner, Eigenvalues of certain operators on matrices, Comm. Pure Appl. Math. 14 (1961), 155-156.

Stevens Institute of TeChNOLOGY 